\title{
MAXIMUM LIKELIHOOD PROPAGATION-DELAY ESTIMATION IN UNKNOWN CORRELATED NOISE USING ANTENNA ARRAYS: APPLICATION TO GLOBAL NAVIGATION SATELLITE SYSTEMS ${ }^{1}$
}

\author{
G. Seco Granados, J. A. Fernández Rubio \\ Signal Theory and Communications Department, Universitat Politècnica de Catalunya (UPC) \\ Campus Nord UPC. Módulo D-5. Jordi Girona,1-3. 08034 Barcelona, SPAIN \\ e-mail: \{gonzalo or juan\}@gps.tsc.upc.es
}

\begin{abstract}
A.BSTRACT
The problem of estimating the propagation-delay of a desired signal in the presence of interferences and multipath propagation is addressed. This paper presents the maximum likelihood (ML) propagation-delay estimator for a signal arriving at a sensor array. The novel characteristic herein is that the desired signal impinges on the array with a known steering vector. This fact allows to assume an unknown and arbitrary spatially colored noise.. The Cramér-Rao bound (CRB) for the problem at hand is derived and numerically compared with the variance of the MLE. The MLE is applied to the Global Navigation Satellite Systems, in order to reduce the serious performance deterioration that the interferences and the multipath propagation produce. We show that in presence of coherent reflections of the desired signal the presented estimator is no longer the MLE and becomes biased. However, its bias is much lower than that of other conventional estimators.
\end{abstract}

\section{INTRODUCTION}

Propagation-delay estimation is a key task in diverse applications such radar, sonar and communications. In the Direct-Sequence Spread Spectrum (DS-SS) communication systems the synchronization between the received pseudo-noise (PN) code and the local code has to be as accurate as possible in order to not increase the bit error probability. Other systems that arouse great interest at present and wherein the propagation delay estimation is fundamental, even more than in the communication systems, are the Global Navigation Satellite Systems (GNSS).

Nowadays there is no doubt that GNSS will complement and even replace in a near future all the other positioning and synchronization systems. At the present time there are two global satellite positioning systems: GPS and GLONASS. They are based on the measurement of the distance between the receiver and a set of satellites[1]. These distances are directly obtained from the signal propagation delay between each satellite and the receiver, so it is clear that in a GNSS receiver the only signal-ofinterest (SOI) is the direct signal or line-of-sight signal (LOSS). Another peculiarity of the GNSS is that the receiver is able to know the direction of arrival (DOA) of the direct signal. This is because the receiver can know its position and the satellite one with a certain degree of error that does noi affect the computation of the angle with which the satellite is observed.

GPS and GLONASS transmit direct sequence-spread spectrum (DS-SS) signals. The distance measurement that the receiver obtains from the propagation delay of the PN code is called pseudorange. The distance can be measured using the carrier phase as well. The carrier phases are more accurate than the pseudoranges. However, in the carrier phase receivers it is also necessary to measure accurate pseudoranges, because the more accurate the pseudoranges, the more efficient the methods to fix the integer ambiguity [2].

The measured distances are affected by the errors produced by several phenomena. Probably the most harmful phenomena are the multipath propagation and the interferences, because their effects can not be mitigated by differential techniques [1]

The most widely used technique to measure the pseudorange is the Delay Locked Loop (DLL) [3]. As the performance of the DLL degrades in presence of multipath propagation, other techniques mono-sensor have been proposed, such the NarrowSpacing DLL [3] and the Multipath Estimating DLL [4]. Neither of these techniques can combat the interferences.

Some techniques to measure the pseudorange using antenna arrays have already been investigated and it is clear that the multi-sensor techniques are the most effective ones to reduce the errors introduced by the interferences and the multipath propagation. An overview of these techniques can be found in [5] together with an iterative method for the propagation-delay estimation and array beamforming that does not need a previous DOA estimation stage.

The purpose of these paper is to present the maximum likelihood (ML) propagation delay estimator for a signal received by an array of sensors. The knowledge of the desired signal DOA allows to assume an unknown correiated noise, which models the thermal noise and all other interferences and jamming. We shall apply the MLE to the GNSS and show that the MLE with antenna arrays is effective to reduce the variance and the bias of the propagation delay estimates in scenarios with interferences and multipath.

\footnotetext{
${ }^{1}$ This work has been partially supported by a grant of the Generalitat de Catalunya and by the National Research Plan of Spain CICYT under grant TIC96-0500-C10-01
} 


\section{ML PROPAGATION-DELAY ESTIMATION}

\subsection{Problem formulation}

Consider an array of $N$ sensors arranged in an arbitrary geometry and having arbitrary responses. The received complex $\mathrm{N}$-vector is modeled as

$$
\mathbf{x}(t)=\alpha_{o} \mathbf{a}_{o} s\left(t-\tau_{v}\right)+\mathbf{n}(t)
$$

where $\mathbf{n}(t)$ is the additive noise, $s(t)$ is an arbitrary and known narrow-band signal-of-interest, $\mathbf{a}_{\mathrm{o}}$ is the known steering vector for the SOI, and $\alpha_{o}, \tau_{o}$ are the unknown complex amplitude and propagation delay associated with the SOI. Let $t_{1}, \ldots, t_{K}$ denote the $K$ time instants at which the snapshots are taken. The sampled data can then be expressed as

$$
\mathbf{X}=\alpha_{o} \mathbf{a}_{o} \mathbf{s}^{\tau}\left(\tau_{o}\right)+\mathbf{N}
$$

where

$$
\begin{aligned}
& \mathrm{X}=\left[\mathbf{x}\left(t_{1}\right) \cdots \mathbf{x}\left(t_{x}\right)\right], \mathbf{N}=\left[n\left(t_{1}\right) \cdots n\left(t_{K}\right)\right] \\
& \mathrm{S}\left(\tau_{o}\right)=\left[s\left(t_{1}-\tau_{o}\right) \cdots s\left(t_{m}-\tau_{o}\right) \cdots s\left(t_{K}-\tau_{o}\right)\right]^{T}
\end{aligned}
$$

The noise vector is assumed to be a zero-mean complex Gaussian vector with unknown covariance matrix $Q$, temporally white and independent of the SOI.

$$
E\left\{\mathbf{n}\left(t_{m}\right) \mathbf{n}^{H}\left(t_{k}\right)\right\}=\mathrm{Q} \delta_{m, k}
$$

where $(.)^{\mathrm{H}}$ denotes complex conjugate transpose. The matrix $\mathbf{Q}$ models both thermal noise and all other interferences and jamming, assuming that they are independent of the desired signal. In an actual scenario the noise and interferences may not be temporally white. Nevertheless, it is shown in section 3 that there is a very slight performance degradation in this case.

Given the model described above, the problem addressed in this paper may be stated as follows: Given a collection of data $\mathbf{X}$ as defined in (2), the vector $\mathbf{a}_{0}$ and the signal $s(t)$, estimate the delay $\tau_{o}$, and the amplitude $\alpha_{o}$ and the matrix $\mathbf{Q}$.

\subsection{The MLE for signal with known direction of arrival in unknown correlated noise}

The log-likelihood function of the data takes the form

$$
\begin{aligned}
& L\left(\mathbf{X} ; \tau_{o}, \alpha_{o}, \mathbf{Q}\right)=-K N \ln \pi-K \ln |\mathbf{Q}|- \\
& -\operatorname{tr}\left\{\mathbf{Q}^{-1}\left(\mathbf{X}-\alpha_{o} \mathbf{a}_{o} \mathbf{s}^{T}\left(\tau_{o}\right)\right)\left(\mathbf{X}-\alpha_{o} \mathbf{a}_{o} \mathbf{s}^{T}\left(\tau_{o}\right)\right)^{H}\right\}
\end{aligned}
$$

It can be shown that (5) is maximized (for fixed $\alpha_{o}$ and $\tau_{o}$ ) by

$$
\hat{\mathbf{Q}}=\frac{1}{K}\left(\mathbf{X}-\alpha_{o} \mathbf{a}_{o} \mathbf{s}^{T}\left(\tau_{o}\right)\right)\left(\mathbf{X}-\alpha_{o} \mathbf{a}_{o} \mathbf{s}^{T}\left(\tau_{o}\right)\right)^{H}
$$

If we define the sample correlations as

$$
\begin{aligned}
& \hat{\mathbf{R}}_{x x}=\mathbf{X X} \mathbf{X}^{H} / K \quad \hat{\mathbf{r}}_{x s}\left(\tau_{o}\right)=\mathbf{X s}^{*}\left(\tau_{o}\right) / K \\
& \hat{P}_{s}=\left|\mathbf{s}\left(\tau_{o}\right)\right|^{2} / K \\
& \hat{\mathbf{R}}_{n n}\left(\tau_{o}\right)=\hat{\mathbf{R}}_{x x}-\frac{1}{\hat{P}_{s}} \hat{\mathbf{r}}_{x s}\left(\tau_{o}\right) \hat{\mathbf{r}}_{x s}^{H}\left(\tau_{o}\right)
\end{aligned}
$$

and substituting (6) into (5) and ignoring parameter independent terms yields the concentrated log-likelihood function

$$
\begin{aligned}
& L\left(\mathbf{X} ; \tau_{o,} \alpha_{o}\right)=-\left.K \ln \left|\hat{\mathbf{R}}_{x x}+\hat{P}_{s}\right| \alpha_{o}\right|^{2} \mathbf{a}_{o} \mathbf{a}_{o}^{H}- \\
& -\alpha_{o} \mathbf{a}_{o} \hat{\mathbf{r}}_{x x}^{H}\left(\tau_{o}\right)-\alpha_{o}^{*} \hat{\mathbf{r}}_{x s}\left(\tau_{o}\right) \mathbf{a}_{o} \mid= \\
& =-K \ln \left|\hat{\mathbf{R}}_{n n}\left(\tau_{v}\right)+\left(\alpha_{o} \mathbf{a}_{o}-\frac{\hat{\mathbf{r}}_{x s}\left(\tau_{v}\right)}{\hat{P}_{s}}\right)\left(\alpha_{o} \mathbf{a}_{o}-\frac{\hat{\mathbf{r}}_{x s}\left(\tau_{o}\right)}{\hat{P}_{s}}\right)^{H}\right|
\end{aligned}
$$

Applying the determinant identiry $\left|\mathbf{I}+\mathbf{B}^{\mathrm{H}} \mathbf{C}\right|=\left|\mathbf{I}+\mathbf{C B}^{\mathrm{H}}\right|$ to (9) and taking into account that (8) does not depends on $\alpha_{o}$ it is easy to show that

$$
\hat{\alpha}_{o}=\frac{1}{\hat{P}_{s}} \frac{\mathbf{a}_{o}^{H} \hat{\mathbf{R}}_{n n}^{-1}\left(\tau_{o}\right) \hat{\mathbf{r}}_{x s}\left(\tau_{o}\right)}{\mathbf{a}_{o}^{H} \hat{\mathbf{R}}_{n n}^{-1}\left(\tau_{o}\right) \mathbf{a}_{o}^{H}}
$$

Substituting this value back in (9), developing (8) according with the previous determinant identity and applying the matrix inversion lemma to (8), we get

$$
L\left(\tau_{o}\right)=-K \ln \left(1-\hat{\alpha}_{v} \hat{\mathbf{r}}_{x s}^{H}\left(\tau_{o}\right) \hat{\mathbf{R}}_{x x}^{-\mathrm{t}} \mathbf{a}_{o}\right)
$$

which can be further simplified if the amplitude estimate (10) is expressed in function of $\hat{\mathbf{R}}_{x x}$ instead of $\hat{\mathbf{R}}_{n n}$

$$
\begin{aligned}
& L\left(\tau_{o}\right)=K \ln \left(1+\left(\mathbf{a}_{o}^{H} \hat{\mathbf{R}}_{x x}^{-1} \mathbf{a}_{o}\right)^{-1} f\left(\tau_{o}\right)\right) \\
& f\left(\tau_{o}\right)=\frac{\left|\mathbf{a}_{o}^{H} \hat{\mathbf{R}}_{x x}^{-1} \hat{\mathbf{r}}_{x s}\left(\tau_{o}\right)\right|^{2}}{\hat{P}_{s}-\hat{\mathbf{r}}_{x s}^{H}\left(\tau_{o}\right) \hat{\mathbf{R}}_{x x}^{-1} \hat{\mathbf{r}}_{x s}\left(\tau_{o}\right)}
\end{aligned}
$$

We finally get the maximum likelihood propagation-delay estimator as

$$
\hat{\tau}_{o}=\arg \max _{\tau_{\mathrm{o}}} f\left(\tau_{o}\right)
$$

It can be proved ([6]) that this MLE is consistent and asymptotically statistically efficient. Important characteristics of this estimator are that it can be applied with any array geometry and that it avoids spectral searches, thus overcoming the main drawbacks of the DOA estimation based methods and the decorrelation methods [5].

\subsection{Cramér-Rao Bound}

The Cramér-Rao bound (CRB) is a lower bound on the variance of any non-biased estimator. The variance of the presented MLE tends asymptotically (when the number of samples $K$ tends towards infinity) towards the CRB. Considering the loglikelihood function of (5), the $i j$ th entry of the Fisher information matrix has the form [6]

$$
\begin{aligned}
& \{F I M\}_{i j}=K \operatorname{tr}\left\{\mathrm{Q}^{-1} \mathrm{Q}_{i}^{\prime} \mathrm{Q}^{-1} \mathrm{Q}_{j}^{\prime}\right\}+ \\
& +2 \operatorname{Re}\left\{\left(\mathbf{a}_{o}^{H} \mathrm{Q}^{-1} \mathbf{a}_{o}\right)\left(\left(\alpha_{o} \mathbf{s}\left(\tau_{o}\right)\right)^{H}\right)_{i}^{\prime}\left(\alpha_{o} \mathbf{s}\left(\tau_{o}\right)\right)_{j}^{\prime}\right\}
\end{aligned}
$$

where $(.)_{i}$ stands for the derivative with respect to the $i$ th real unknown of the log-likelihood function. The FIM is block diagonal since $Q$ does not depend on $\alpha_{o} \tau_{o}$ or vice versa. If we only consider the delay, the modulus and phase of the amplitude the corresponding block of the FIM is 


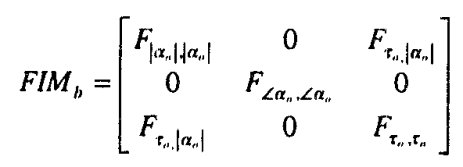

and

$$
C R B_{b}=F I M_{b}^{-1}
$$

where

$$
\begin{aligned}
& F_{\left|\alpha_{n}\right|+\alpha_{o} \mid}=2 K \hat{P}_{s} \mathbf{a}_{o}^{H} \mathbf{Q}^{-1} \mathbf{a}_{o} \\
& F_{\angle \alpha_{o}, \angle \alpha_{o}}=2 K\left|\alpha_{o}\right|^{2} \hat{P}_{s} \mathbf{a}_{o}^{H} \mathbf{Q}^{-1} \mathbf{a}_{o} \\
& F_{\tau_{o}, \tau_{n}}=2\left|\alpha_{o}\right|^{2}\left|\mathbf{s}\left(\tau_{o}\right)\right|^{2} \mathbf{a}_{o}^{H} \mathbf{Q}^{-1} \mathbf{a}_{o} \\
& F_{\tau_{n}\left|\alpha_{o}\right|}=2\left|\alpha_{o}\right| \operatorname{Re}\left\{\mathbf{s}^{H}\left(\tau_{o}\right) \dot{\mathbf{s}}\left(\tau_{o}\right)\right\} \mathbf{a}_{o}^{H} \mathbf{Q}^{-1} \mathbf{a}_{o}
\end{aligned}
$$

with

$$
\left\{\dot{\mathrm{s}}\left(\tau_{c}\right)\right\}_{i}=-\left.\frac{d s(t)}{d t}\right|_{t_{i}-\tau_{n}}
$$

\section{SIMULATION RESULTS}

This section examines the performance of the MLE. Although the formulation has been absolutely general, we are going to apply it to the problem of estimating the delay of the line-ofsight signal received from a GPS satellite. Each GPS satellite transmits several DS-SS signals, but civil users have only access to the carrier spread by the C/A code, with a chip rate equal to $1.023 \mathrm{Mchips} / \mathrm{s}$. This carrier is called L1 and it is also modulated by the 50 bits/s navigation message. The received signal power is well below the noise power, but passing the output of each antenna through a matched filter to the C/A code yields a "finger" for each multipath arrival every bit period. Each finger is the autocorrelation of the $\mathrm{C} / \mathrm{A}$ code, with a duration roughly equal to 2 chips. These "fingers" are above the noise level thanks to the $43 \mathrm{~dB}$ processing gain, however they can be below the interferences. As the use of an antenna array in the receiver should be useful to combat the interferences and the multipath, but not necessarily the noise which is enough attenuated in the despreading process, it is necessary to apply the MLE at the output of each antenna matched filter. Therefore, the signal $s(t-$ $\left.\tau_{o}\right)$ in (1) represents in cur case the "fingers" corresponding to the line-of-sight signal.

In the simulations below we are going to consider two performance measures: the variance and the bias of the estimator. In all the cases the array is assumed to be linear with uniform half-wavelength spacing and the signal power to noise spectral density ratio is $40 \mathrm{~dB}-\mathrm{Hz}$, which yields a SNR equal to $23 \mathrm{~dB}$ after the despreading. The line-of-sight signal DOA is $30^{\circ}$ and the noise is spatially white. The RMS (root mean square) delay estimation errors presented below are obtained from 200 Monte Carlo simulations.

\subsection{Delay estimation variance}

In the first experiment, we investigate the variance of the MLE as the number of samples $K$ is varied. The results are plotted in Figure 1 for the samples being taken in 1 and 2 fingers. Rough previous synchronization with an error up to a tenth of a chip is assumed. The first thing that stands out from this figure is the nearly unappreciable improvement when taking the samples in 2 fingers. Also the RMSE does not attain the CRB. For small numbers of samples the RMSE decreases with the number of samples, but later it tends to a constant value. The reason for these behaviors is that the noise (at the output of the matched filter) is not temporally white as it was considered in section 2 .

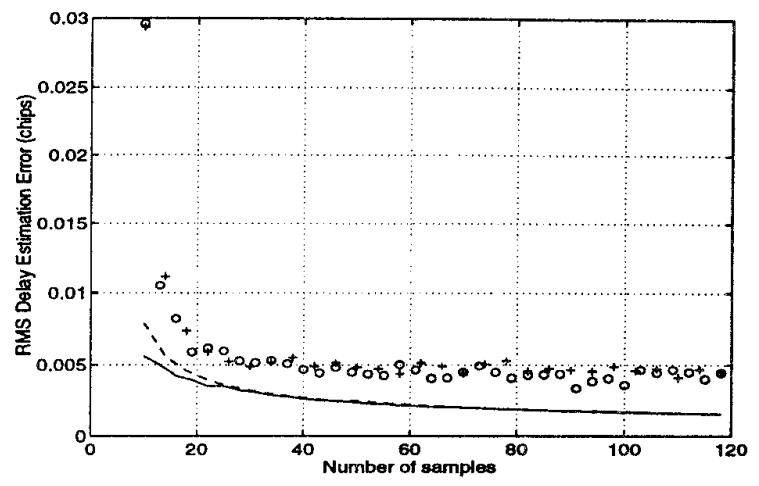

Figure 1. $N=10$ antennas. The direct signal and noise are received. Solid line is for the CRB with 1 finger and dashed line for the CRB with 2 fingers. ' 0 ' and ' + ' are for the RMSE with 1 and 2 fingers, respectively.

In the second experiment (Figure 2), we compare the RMSE against the CRB when an interference is received in function of its power. Again the RMSE does not attain the CRB due to the fact that the interference is not temporally white. However, note that the RMSE and the CRB tend to a constant value as the interference power increase. This implies that the receiver can hold up arbitrarily strong interferences using an antenna array.

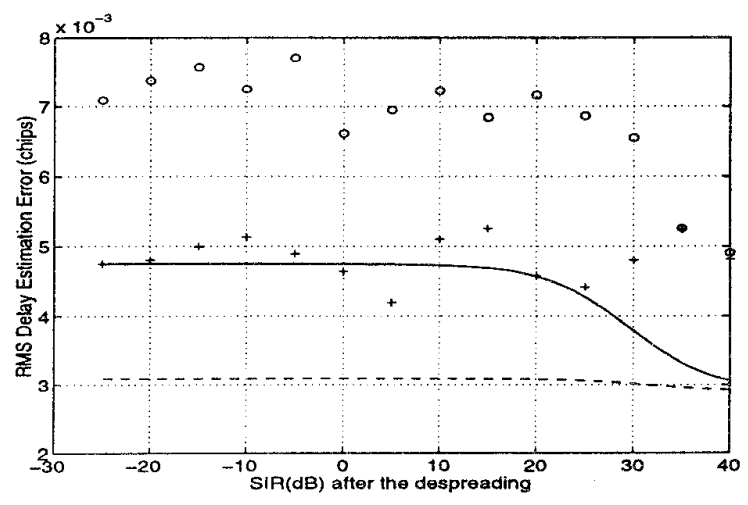

Figure 2. $N=10$ antennas, $K=35$ samples in one finger. In solid line the CRB when the interference arrives from $35^{\circ}$ and ' $o$ ' is the RMSE in this case. The dashed line and ' $t$ ' are the CRB and RMSE when the interference arrives from $40^{\circ}$.

In the third experiment, the effect of the number of antennas on the CRB and the RMSE is analyzed. The results are illustrated in Figure 3 . As predicted by theory, the CRB and the RMSE are reduced when the number of antennas increases. Furthermore, 
for a small number of antennas the higher the interference, the more antennas are needed to achieve the same RMSE.

\subsection{Delay estimation bias}

In presence of coherent multipath the received signal can not be modeled as in (1), where it was divided in the direct signal term and in an additional term incorrelated with the former. Therefore the estimator (13) is no longer the MLE and it suffers from a certain bias. Figure 4 illustrates the asymptotic maximum bias produced by an single reflection in the estimator at hand and in a conventional DLL[3]. The bias depends on the angular separation between the direct signal and the reflection, however, in all the cases the bias of the proposed estimator is much lower than that of the DLL.

In the final example, we investigate the effect of increasing the number of antennas. As expected, Figure 5 shows how increasing the number of antennas is effective for reducing the bias.

\section{CONCLUSIONS}

We have presented the maximum likelihood propagation-delay estimator for a desired signal with known DOA arriving at a sensor array in presence of unknown and arbitrary spatially colored noise, which has been considered temporally white. This estimator is applied to measurement of the propagation delay of the GPS signals. The estimation variance is slightly greater than the CRB, due to the non-white noise and interferences in the actual scenario. In presence of coherent multipath propagation the bias of the investigated estimator is much lower than that of the conventional methods. Therefore, it has been proved that the use of antenna arrays together with the ML propagation-delay estimator allows to greatly reduce the effects of the interferences and the multipath, which are the most harmful sources of errors in GNSS.

\section{REFERENCES}

[1] Wells, David. Guide to GPS positioning. Canadian GPS Associates, 1986-1987.

[2] Park, C., Kim, I., Gyu Lee, J., Jee G. "Efficient technique to fix GPS carrier phase integer ambiguity on-the-fly". IEE Proceedings in Radar, Sonar and Navigation, 144(3):148155, June 1997.

[3] Van Nee, R.D.J. "Spread-Spectrum Code and Carrier Synchronization Errors caused by Multipath and Interference", IEEE Trans. on AES, 29(4): 1359-1365, October 1993.

[4] Saarnisaari, H. "ML time delay estimation in a multipath channel", ISSSTA'96, pp. 1007-1011, Mainz, Germany.

[5] Seco Granados G., Fernández Rubio J., "Multipath and Interference Errors Reduction in GNSS by joint pseudorange measurement and array beamforming". GNSS' 97. First European Symposium on Global Navigation Satellite Systems, pp. 606-614, 21-25 April 1997. Munich, Germany.

[6] Kay, S.M. Fundamentals of Statistical Signal Processing. Estimation Theory. Prentice-Hall 1993.

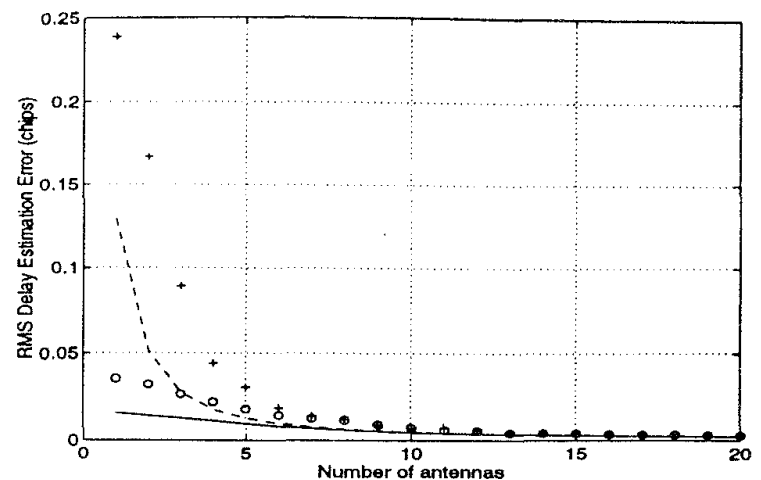

Figure 3. Interference DOA $35^{\circ}$ and $K=35$ samples in one finger. Solid line and ' $o$ ' for the CRB and RMSE when $\mathrm{SIR}=20 \mathrm{~dB}$ (after despreading). Dashed line and ' $t$ ' for the CRB and RMSE when $S I R=0 \mathrm{~dB}$ after despreading.

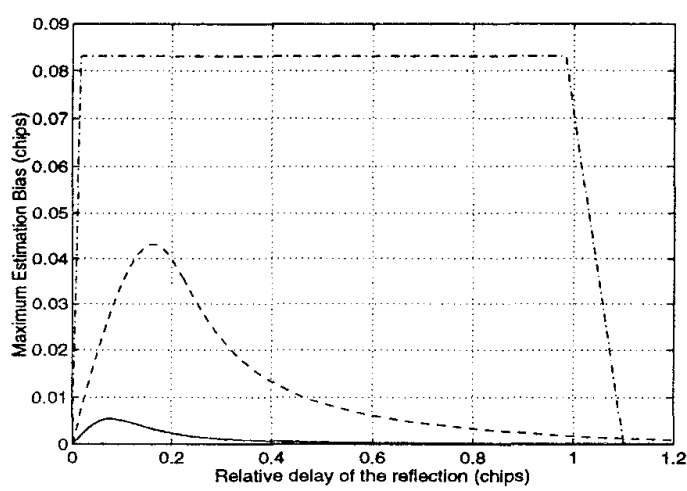

Figure 4. The reflection is $1.6 \mathrm{~dB}$ below the direct signal. The dashdotted line represents the bias of a DLL with early-late spacing 0.2 chips. The solid and dashed lines correspond to the MLE using 8 antennas and the reflection DOA being $-40^{\circ}$ and $35^{\circ}$, respectively.

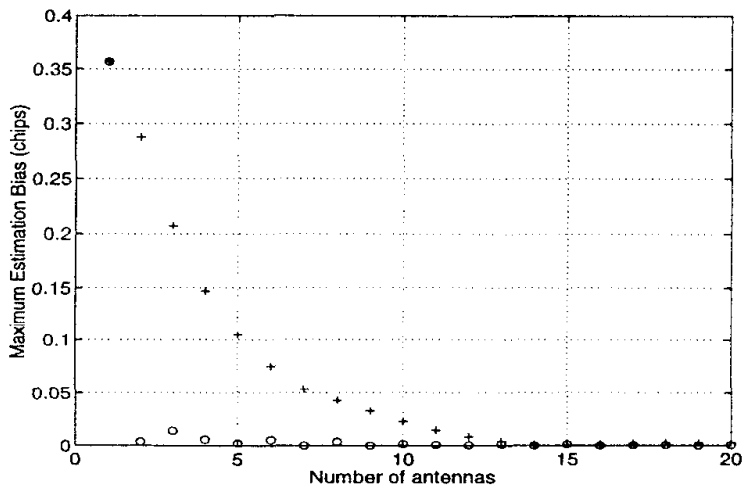

Figure 5. One reflection $1.6 \mathrm{~dB}$ below the direct signal and delayed 0.15 chips. The reflection DOA is $-40^{\circ}$ for 'o' and $35^{\circ}$ for ' + '. 